\title{
Progress in calibrating an X-ray spectrometer
}

\section{Ib Sørensen}

Information on the X-ray fluorescence spectrometry carried out at the Survey has been briefly presented elsewhere (Sørensen, 1975). This note describes the progress made in developing a calibration and matrix correction procedure.

Like all other instrumental methods for elemental analysis, X-ray spectrometry is dependent on material of known composition for calibration, since this is the only way to get information about which function $\mathrm{F}$ exists between the concentration $C_{i}$ and the measured intensity $E_{i}$ caused by the element $i$, say

$$
E_{i}=F\left(C_{i}\right)
$$

For several years chemically analysed samples were used as calibration tools, but it was not long to establish that the term 'known composition' of a natural rock powder is rather uncertain. The theory and practice of X-ray spectrometry has been studied in a lot of detail and the precision can in fact be very high. It is therefore rather unsatisfactory for the calibration accuracy to be dependent on natural samples of doubtful composition.

A method employed in the Department of Earth Sciences of the University of Leeds, England, uses synthetic standards prepared in the same way as the samples for analysis, by melting fixed amounts of pure oxides with sodium tetraborate. Details about the matrix corrections make it impractical to use this method at GGU. During 1975 we have therefore developed a calibration and matrix correction procedure which fits well to the computer controlled Siemens SRS spectrometer and the programmes LINQUA/MATKOR. This method makes a compromise between reasonable accuracy and simplicity of operation within the limits of programme possibility. For instance we cannot make background corrections automatically such as required for magnesium and phosphorus. Prior to a fuller description of the method a brief outline is presented here.

\section{Calibration}

In calculating the concentrations $C_{E, i}$ from measured XRF-intensities $E_{i}$ the equation $C_{E, i}$ $=A+B \cdot E_{i}+C \cdot E I^{2}$ is used. The calibration - which is the determination of the constants $A, B$ and $C$-is performed automatically by setting the programme LINQUA in a calibrating position and measuring the standards of known concentration values. These standards are prepared in the same way as the unknown samples by melting carefully weighed pure oxides in calculated amounts with sodium tetraborate. They.contain only one element oxide besides sodium tetraborate and the concentration values are norm numbers, i.e. 10, 12.5, 16, $20,25,31.5,40,50,63,80$ and $100 \%$. In most cases it is sufficient to use only the values 10 , 16,25 and 40.

Although the high and the low standards will show different mass-absorption, the effect of this will be incorporated in the non-linear second degree term of the concentration equation. In general the constants $C$ are very small. These synthetic monoelement standards, termed SYMES, are free from interelement effects and accordingly the true concentration $C_{i}$ is equal to $C E, i$. 


\section{Matrix correction}

If more than one element is present, which is the case in unknown samples, interelement effects occur. We correct for this by using the equation $C_{i}=C_{E, i}\left(1+\Sigma K_{J} . C_{j}\right)$. Again $C_{i}$ is the true concentration of element $i, C_{E, i}$ the concentration calculated from measured intensity $E_{i}$, and $K_{j}$ interelement coefficients for element $j$ in concentration $C_{j}$.

The coefficients $K_{j}$ are determined experimentally by measuring synthetic binary, ternary and in single cases quaternary standards prepared as the SYMES by melting carefully weighed off pure oxides with sodium tetraborate. We never use more complicated standards than quaternary. The matrix equation gives us directly the $K_{j}$ values for binary standards, and in the case of ternary or quaternary standards it gives us a number of simple linear equations. They can be solved with a small pocket calculator. The system of equations is normally over determined, and therefore yields several possible solutions. From these a selection is made within the limits of experimental error so that the final values both in magnitude and sign are in accordance with what would be expected from theoretical considerations.

\section{Conclusions}

The system has been tested with several hundred samples of various compositions, including international geochemical standard reference materials. Besides excellent accuracy the system has a high degree of flexibility: It is possible for instance to analyse an iron-titanium bearing rock with $75 \% \mathrm{Fe}_{2} \mathrm{O}_{3}$ and $20 \% \mathrm{TiO}_{2}$ with the same set of calibration and matrix correction constants as used for silicate rocks of normal composition.

In this way it is possible to use XRF as a method of elemental analysis completely independent of other analytical methods, being dependent only on fundamental chemical and physical constants, the analytical balance and on skill.

\section{Reference}

Sørensen, I. 1975: X-ray fluorescence spectrometry at GGU. Rapp. Grønlands geol. Unders. 75, 16-18. 